\title{
EVALUATION OF FORECASTS IN AR MODELS WITH OUTLIERS
}

\author{
Regina Elsebach, Universität der Bundeswehr Hamburg
}

An important problem in time series analysis is forecasting when outliers are present. Outliers are modelled as "additive" or "innovational" with respect to a basic time series process. Since it is a main characteristic of outliers that their occurence is not predictable, the aim of any forecast is the prediction of how the series will develop under the basic process without outliers. Ex-post analysis of forecasts should pay attention to the fact that extreme forecast errors can be caused by outliers, not by the basic process itself. Such extreme errors should not dominate the ex-post evaluation.

Based on the well-known AR model of order $p$,

$$
X_{t}-\mu=\sum_{i=1}^{p} \phi_{i}\left(X_{t-i}-\mu\right)+\varepsilon_{t}, \varepsilon_{t}-\mathbf{N}\left(0, \sigma^{2}\right) \text { iid, }
$$

the effect of additive and innovational outliers on the standard MSE-optimal 1inear $\tau$-step-ahead foiecast (with $(p, \tau-1):=\min \{p, \tau-i\})$

$$
\hat{x}_{t}(\tau)=\sum_{i=1}^{(p, \tau-1)} \phi_{i} \hat{x}_{t}(\tau-i)+\sum_{i=(p, \tau-1)+1}^{p} \phi_{i} x_{t+\tau-i}+\mu\left(1-\sum_{i=1}^{p} \phi_{i}\right)
$$

and the resulting forecast errors will be examined for $\tau=1$. Two alternative methods to evaluate forecasts based on the realized errors - robust generalizations of the standard mean squared (MSE) and mean absolute error (MAE) called MRSE resp. MRAE are presented, and their behavior is visualized by a simulation study.

Especially in the case of additive outliers, these methods compare favourably to the standard MSE and MAE. Given some competing forecasts based on robust and non-robust estimates of the parameters of the underlying AR model, the MSE (and to a lesser extent also the MAE) very often identifies a forecast based on very poor estimates as best. This is considered to be inadequate. MRSE and MRAE are much better suited to detect those forecasts which are based on the true underlying AR parameters. 